\title{
Health care workers causing large nosocomial outbreaks: a systematic review
}

Lisa Danzmann ${ }^{1}$, Petra Gastmeier ${ }^{2}$, Frank Schwab² and Ralf-Peter Vonberg ${ }^{\text {** }}$

\begin{abstract}
Backgrounds: Staff in the hospital itself may be the source of a nosocomial outbreak (NO). But the role of undetected carriers as an outbreak source is yet unknown.

Methods: A systematic review was conducted to evaluate outbreaks caused by health care workers (HCW). The Worldwide Outbreak Database and PubMed served as primary sources of data. Articles in English, German or French were included. Other reviews were excluded. There were no restrictions with respect to the date of publication. Data on setting, pathogens, route of transmission, and characteristics of the HCW was retrieved. Data from large outbreaks were compared to smaller outbreaks.

Results: 152 outbreaks were included, mainly from surgery, neonatology, and gynecology departments. Most frequent corresponding infections were surgical site infections, infection by hepatitis B virus, and septicemia. Hepatitis B virus (27 NO), S. aureus (49 NO) and S. pyogenes (19 NO) were the predominant pathogens involved. 59 outbreaks (41.5\%) derived from physicians and 56 outbreaks (39.4\%) derived from nurses. Transmission mainly occurred via direct contact. Surgical and pediatric departments were significantly associated with smaller outbreaks, and gynecology with larger outbreaks. Awareness of carrier status significantly decreased the risk of causing large outbreaks.
\end{abstract}

Conclusions: As NO caused by HCW represent a rare event, screening of personnel should not be performed regularly. However, if certain species of microorganisms are involved, the possibility of a carrier should be taken into account.

Keywords: Nosocomial outbreak, Staff, Personnel, Health care workers, Health care associated infection

\section{Background}

Healthcare associated infections (HCAI) are infections that are acquired as a result of healthcare interventions. Most HCAI occur sporadically only. However, epidemics of HCAI may also take place and are than called a nosocomial outbreak (NO). NO always represent extremely frightening incidents, but may still affect any medical department at any time in principle. Once a NO has been recognized as such, an outbreak investigation is usually initiated in order to discover its source. Doing so, the outbreak's source may get traced to a particular single health care worker $(\mathrm{HCW})$ in some of the cases. If now a HCW, in fact, happens to be the most likely or proven cause of pathogen spread to patients and nosocomial

\footnotetext{
* Correspondence: Vonberg.Ralf@MH-Hannover.DE

'Institute for Medical Microbiology and Hospital Epidemiology, Hannover

Medical School, Carl-Neuberg-Str. 1, Hannover D-30625, Germany

Full list of author information is available at the end of the article
}

infections, all of a sudden several uncertainties and questions arise, for example: Was the $\mathrm{HCW}$ aware of his/her carrier status? Were occupational physicians of the hospital wrong at evaluating the risk of transmission from a colonized or infected HCW? Are there obvious breaches in infection control measures? Are there specific risk factors that may have contributed to an extraordinary high transmission rate?

There are several reports of NO that started from a HCW. However, every report by itself is very much influenced by the local situation. Thus, one can hardly generalize the experiences from a single NO description. Only a systematic evaluation of a large number of outbreak reports will provide a less biased assessment of data.

This article presents findings of a systematic analysis of all kinds of published NO that were caused by HCW.

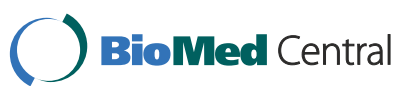

(c) 2013 Danzmann et al.; licensee BioMed Central Ltd. This is an Open Access article distributed under the terms of the Creative Commons Attribution License (http://creativecommons.org/licenses/by/2.0), which permits unrestricted use, distribution, and reproduction in any medium, provided the original work is properly cited. 
By this, we will provide HCW characteristics and factors that may dramatically facilitate pathogen spread.

\section{Methods}

\section{Retrieval strategy}

We conducted a systematic review of the medical literature. Search of the literature was performed on June $23^{\text {rd }} 2010$. In order to find appropriate descriptions of $\mathrm{NO}$ we first of all made a data request for outbreaks whose source was "personnel" in the Outbreak Database (www.outbreak-database.com). This is an internet-based, worldwide database for NO continuously updated by the Institute for Hygiene and Environmental Medicine, Charité - University Medicine Berlin (Germany) currently containing over 2,900 outbreaks [1]. In a second step we supplemented our data by a PubMed search on September $3^{\text {rd }} 2010$, consisting of the following search terms [("nosocomial") AND ("outbreak" OR "epidemic") AND ("personnel" OR "staff" OR "health care worker")]. Finally, we looked through the references of the articles which had been included by then to complete the search.

\section{Exclusion and inclusion criteria}

Besides staff in the hospital being the outbreak's source, inclusion criteria were as follows: articles written in English, German or French language. Reviews were excluded to avoid bias. There were no restrictions with respect to the date of publication.

\section{Extracted data}

(a) We extracted data on the setting (time, country, medical department), patients (type of infections, causative agent, number of fatal cases), (b) characteristics about the staff that caused the outbreak (kind of profession, infection among themselves vs. colonization only, route of transmission, awareness of their own positive carriage status of pathogen, compliance to hand hygiene, length of work experience), and (c) the infection control measures implemented to terminate the outbreak (screening of patients and/or staff, disinfection and sterilization procedures, isolation of patients, application of antimicrobial substances, education of personnel, use of protective clothing, improvement in hand hygiene, closure of ward, sampling of medical devices and/or the environment, changes in patient-staff ratio, use of vaccinations, no implementing of any infection control measures at all). Data was primarily extracted by one author (L.D.) and than independently cross-checked by another author (R.P.V.). A third author (P.G.) got involved in the case of disagreement between the other two authors.

\section{Large outbreaks}

NO were defined as large outbreaks (LO), when the number of affected patients was equal or greater than the median number of patients in all NO.

\section{Statistics}

In the descriptive statistic, rates were calculated for LO stratified by the following risk factors: Type of department, age groups (neonates, infants, children, adolescents, adults, seniors), type of ward (intensive care, peripheral ward, outpatients clinic, operating theatre), outbreak happening before or after 1989 (to identify the trend of the past years and as this was the time when vaccination against hepatitis B virus (HBV) was already recommended in several countries, e. g. the USA [2,3]), causative agent, specific characteristics of the source, and infection control measures. For univariate analysis (contingency tables), the Fisher exact test was used, with a significance level of 0.05 . Multiple logistic regression analysis was performed with stepwise variable selection to detect $\mathrm{LO}$ with the parameters mentioned above. We set $\mathrm{p} \leq 0.05$ for entering a parameter into the model. We used the commercial statistical package Statistical Analysis System (SAS, Version 9.2, the SAS Institute Inc., Cary, NC, USA) for the analysis. Significance level was set at 0.05 .

\section{Results}

Included articles

At the time point of retrieval the Outbreak Database comprised 222 articles in which HCW were filed as the outbreak's source. 116 descriptions thereof met the inclusion criteria. Outbreaks in facilities other than hospitals (e. g., nursing homes) got excluded, as did NO with a HCW being suspected only (but not proven) the true source. Altogether $5 \%$ of all outbreaks published in the Outbreak Database were included. The PubMed search (609 hits) finally came up with 5 more NO reports. 31 additional reports were found via the search of reference lists. Thus, this systematic review is based on overall $152 \mathrm{NO}$ descriptions caused by HCWs (Figure 1). The complete list of articles can be retrieved as an Additional file 1. It

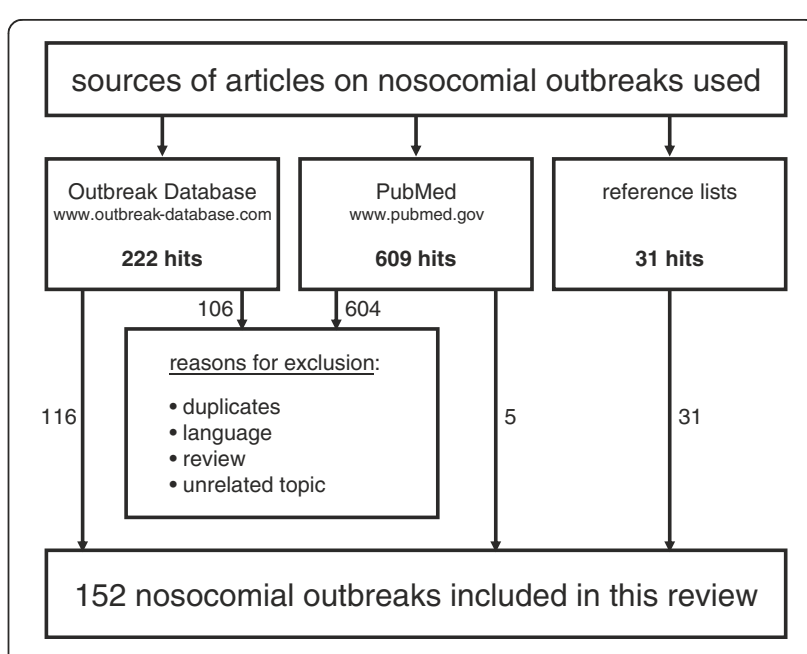

Figure 1 Retrieval of articles. 
consists of 140 articles (9 authors described 2 NO and another author described 4 different NO within one single article).

\section{Univariate analysis \\ Setting}

The 152 NO took place in 26 countries, mainly in the US (67 NO), the UK (29 NO), and France (8 NO). The time frame of occurrence was 1958 through 2006. NO lasted between 1 and 287 weeks (mean: 28.4; median: 10.5). 9 NO showed a polyphasic progress with more than one peak in the epidemic curve.

\section{Patients}

Overall, 1,449 patients (thereof at least 51 fatal cases) were affected ranging from 1 to 75 patients per NO (mean: 9.5; median: 7.0). Thus, a number of patients $\geq 7$ divided 76 large outbreaks (LO) from 76 other smaller NO.

\section{Medical departments and type of ward}

As shown in Table 1, departments of surgery (76 NO), neonatology (41 NO), gynecology (20 NO), pediatrics (9 NO), and internal medicine (2 NO) were most often affected by NO derived from HCW. LO were less often noticed in surgical departments (42.1\%) compared to other types of medical departments (59.1\%). The same applies to pediatric departments where the proportion of LO was $11.1 \%$ only vs. $53.2 \%$ in other medical fields. In $2 \mathrm{NO}$ no specific medical department was mentioned.

Transmission of most $\mathrm{NO}$ occurred in operating theatres (63 NO) and on peripheral wards (59 NO). The risk of LO via operating theatres was slightly decreased ( $41.3 \%$ vs. $57.5 \%$; $=0.068$ ) but failed to reach statistical significance.

\section{Infection types}

Besides several cases of colonization, there were also at least 960 documented cases of nosocomial infections among the 1,449 patients. The most frequent nosocomial infections were surgical site infections (SSI; 256), HBV infections (212), septicemia (67), gastroenteritis (42), hepatitis $\mathrm{C}$ virus infections (HCV) (21), urinary tract infections (20), and meningitis (13).

\section{Causative agent}

Transmission of bacteria occurred in 108 of the 152 NO. Viral spread (34 NO) and fungi (10 NO) were less often the causative agents. Table 1 shows a detailed distribution of the most frequently detected microorganisms in $\mathrm{NO}$ caused by HCW which were S. aureus (49 NO), HBV (27 NO), and Group A streptococci (19 NO). Regardless of the number of patients (LO vs. other NO), spread of the pathogen via direct contact was the main route of transmission, followed by droplets and airborne transmission.
Although the source was known (HCW), the specific way of transmission still remained unknown in $8.6 \%$ of all NO.

\section{Characteristics of index personnel}

Despite their rather low proportion among hospital staff, physicians were the group of professionals who caused most outbreaks (59 NO [41.5\%], thereof $30 \mathrm{NO}$ caused by surgeons) compared to $56 \mathrm{NO}(39.4 \%)$ caused by nurses (Figure 2). Other professions frequently involved were technical staff ( $9 \mathrm{NO})$, kitchen staff (5 NO), and midwives (5 NO).

A total of 73 of the spreading HCWs were colonized only and 70 others were themselves infected by the pathogen (as described in the outbreak reports) before spread to their patients took place (Table 1). If the index HCW was colonized only (but not infected) large outbreaks were more common (58.9\%) compared to outbreaks where the index person also showed signs and symptoms of infection (43.0\%). Especially HCW suffering from blood-borne infections were less likely to become a super spreader (33.3\% vs. $54.4 \%$; Table 1 ).

Data on work experience was available for few outbreak events only. However, a subgroup analysis of $\mathrm{HCW}$, stratified by the time of employment, revealed that work experience of a HCW was neither a predicting nor a protecting factor for being a super spreader later on. In $18 \mathrm{NO}(50.0 \%)$ the HCW had worked for less than 5 years compared to $15 \mathrm{NO}(41.7 \%)$ in which the index HCW had work experience for at least 10 years.

Precise information about the spreading HCW with respect to hand hygiene $(\mathrm{HH})$ was also scarce. Only 37 authors provided information on the previous $\mathrm{HH}$ compliance of the spreading $\mathrm{HCW}$, thereof $\mathrm{HH}$ compliance was considered "adequate" in 21 of $37 \mathrm{NO}(56.8 \%)$ but "insufficient" in $16 \mathrm{NO}$ (43.2\%).

Of the 152 spreading HCW only 14 declared that they had previously been aware of their positive pathogen carrier status; 10 of these $14 \mathrm{HCW}$ were affected by some kind of viral hepatitis. However, the HCW's awareness of harboring the pathogen significantly lowered the risk of subsequently causing large outbreaks (21.4\% vs. $52.5 \%$; $\mathrm{p}=0.045)$.

\section{Infection control measures}

In order to provide a complete overview on outbreaks caused by $\mathrm{HCW}$, corresponding measures were also recorded in this systematic review. Screening for pathogen carriage were the measures carried out most frequently as seen in Figure 3. Furthermore, there were several infection control measures that were more often performed in larger outbreaks such as changes in disinfection and/or sterilization processes (52 vs. $19 \mathrm{NO}$ ), enforcement of hand hygiene compliance (42 vs. $28 \mathrm{NO}$ ), environmental sampling ( 45 vs. $19 \mathrm{NO}$ ), and closure of an entire ward/ unit (17 vs. $5 \mathrm{NO})$. 
Table 1 Risk factors for the occurrence of large outbreaks ( $\geq 7$ patients) as determined by univariate analysis (more than one type may be affected)

\begin{tabular}{|c|c|c|c|c|c|c|c|}
\hline \multirow[t]{2}{*}{ Risk factor } & \multicolumn{3}{|c|}{ Risk factor present } & \multicolumn{3}{|c|}{ Risk factor lacking } & \multirow[t]{2}{*}{ p-value } \\
\hline & \# LO & \# all NO & $\%$ & \# LO & \# all NO & $\%$ & \\
\hline \multicolumn{8}{|l|}{ departments $(n=150)^{*}$} \\
\hline surgery & 32 & 76 & 42.1 & 44 & 74 & 59.1 & 0.036 \\
\hline neonatology & 24 & 41 & 58.5 & 52 & 109 & 47.7 & 0.274 \\
\hline gynecology & 14 & 20 & 70.0 & 62 & 130 & 47.7 & 0.091 \\
\hline pediatrics & 1 & 9 & 11.1 & 75 & 141 & 53.2 & 0.017 \\
\hline internal medicine & 1 & 2 & 50.0 & 75 & 148 & 50.7 & 1.000 \\
\hline other & 11 & 21 & 52.4 & 65 & 129 & 50.4 & 1.000 \\
\hline
\end{tabular}

type of ward $(n=150)^{*}$

$$
\begin{array}{r}
\text { operating theatre } \\
\text { peripheral ward } \\
\text { intensive care unit } \\
\text { outpatient clinic }
\end{array}
$$

age groups $(n=147)^{*}$

$$
\begin{array}{r}
\text { neonates }(\leq 1 \mathrm{~m}) \\
\text { infants }(1 \mathrm{~m}-1 \mathrm{y}) \\
\text { children }(>1-12 \mathrm{y}) \\
\text { adolescents }(13-17 \mathrm{y}) \\
\text { adults }(18-69 \mathrm{y}) \\
\text { seniors }(\geq 70 \mathrm{y})
\end{array}
$$

microorganism $(n=152)$

bacteria
viruses
fungi

species $(n=152)$

S. aureus
hepatitis B virus
S. pyogenes
Candida spp.
P. aeruginosa

transmission $(n=152)$

contact
droplets
airborne
foodborne
unknown

\section{HCW characteristics}

\begin{tabular}{rccccccc} 
colonization only & 43 & 73 & 58.9 & 34 & 79 & 43.0 & 0.054 \\
HWC infected & 31 & 70 & 44.3 & 46 & 82 & 56.1 & 5.193 \\
blood borne infection & 9 & 27 & 33.3 & 68 & 125 & 0.057 \\
aware of carrier status & 3 & 14 & 21.4 & 62 & 118 & 52.5 & 0.045 \\
proper HH compliance & 12 & 21 & 57.1 & 9 & 16 & 56.3 & 1.000 \\
work experience $>5 \mathrm{y}$ & 9 & 18 & 50.0 & 8 & 12 & 66.7 \\
\hline
\end{tabular}

$L O=$ large outbreak ( $\geq 7$ patients affected); $N O=$ nosocomial outbreak; $H C W=$ health care worker; $H H=$ hand hygiene; ${ }^{*}$ more than one type may be affected. 


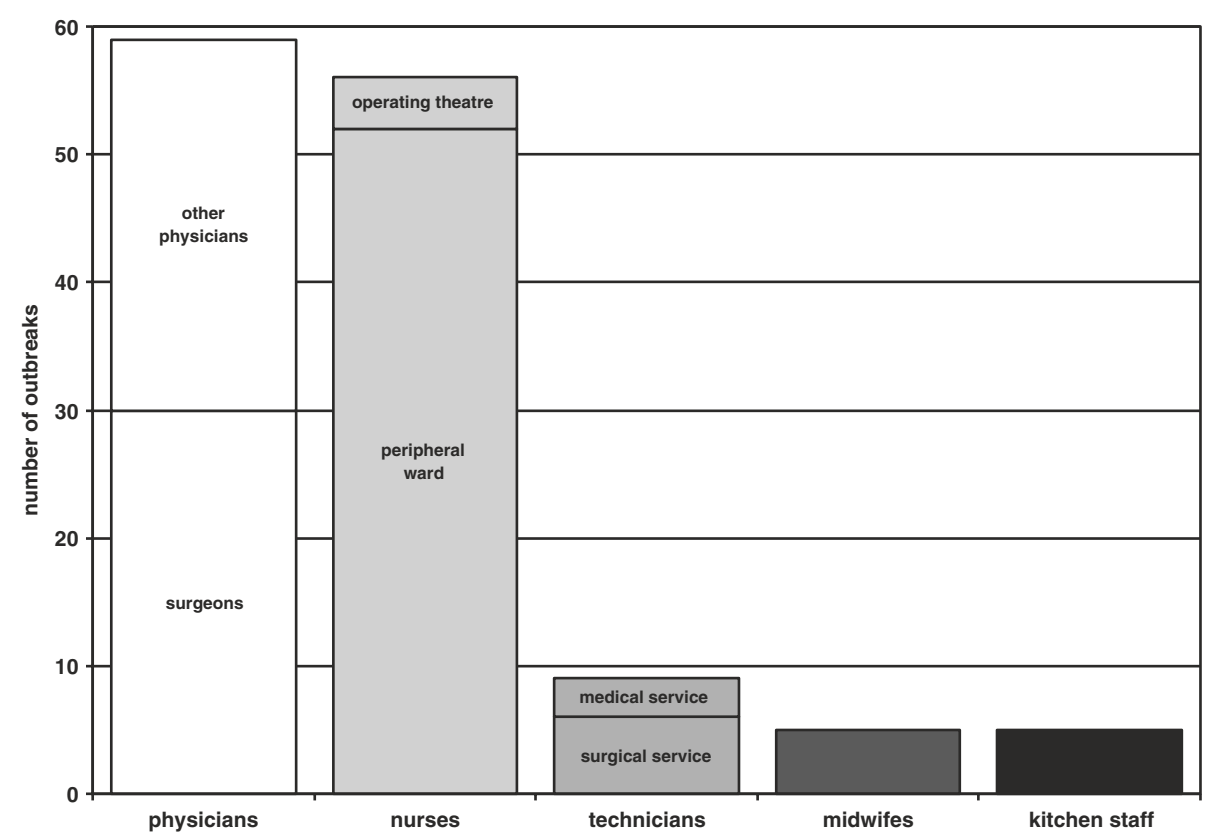

Figure 2 Occupation of health care workers causing nosocomial outbreaks.

\section{Multiple logistic regression analysis}

In order to identify independent factors that increase the risk of a LO, a multiple logistic regression analysis was performed as described above. The remaining characteristics after stepwise variable selection are shown in Table 2.
$\mathrm{HCW}$ in gynecological departments caused significantly more often large outbreaks with an odds ratio $(\mathrm{OR})=$ 6.89 , in contrast to surgical departments $(\mathrm{OR}=0.42)$ and pediatrics $(\mathrm{OR}=0.05)$ where large outbreaks were much less common. In addition, awareness of the $\mathrm{HCW}$ which is carrier of an infectious agent significantly reduced the risk

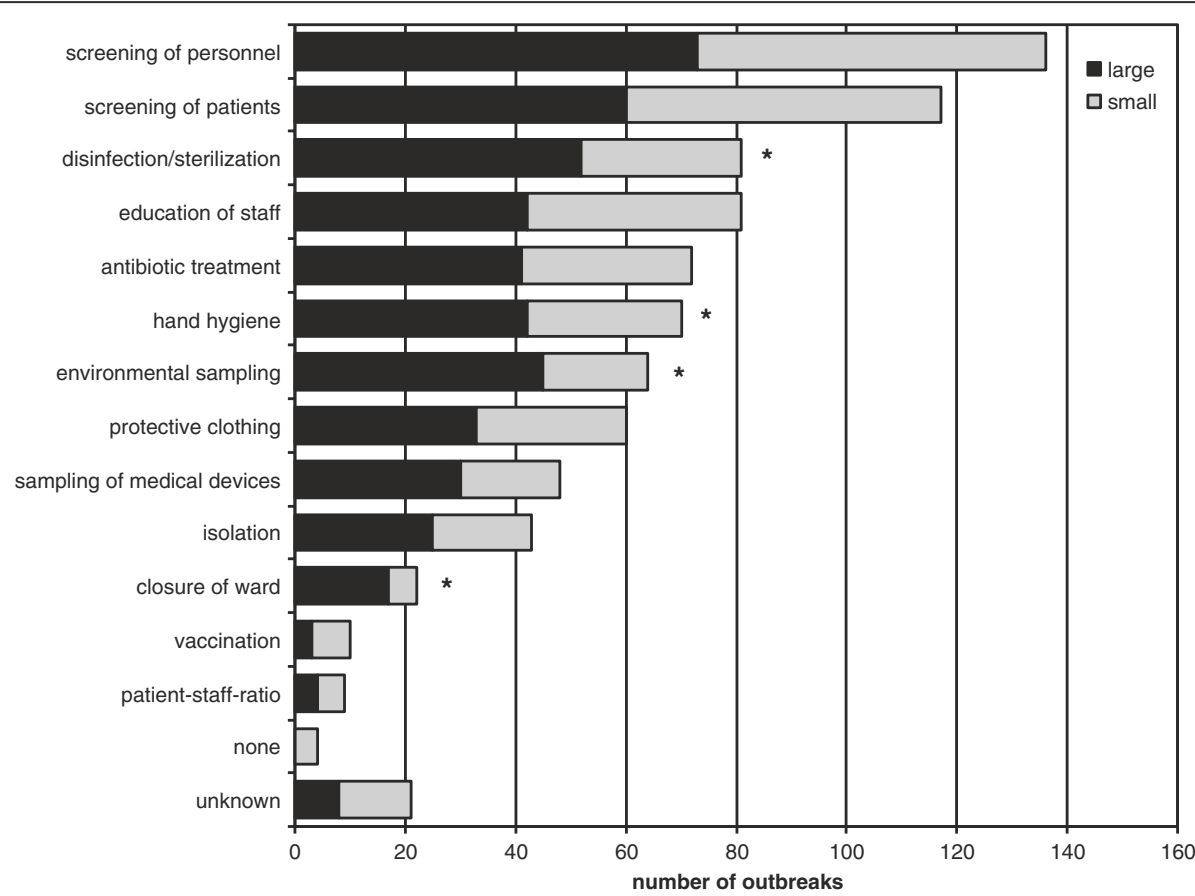

Figure 3 Distribution of infection control measures initiated in large and small outbreaks $(*=$ significant difference). 
Table 2 Independent risk factors for the occurrence of large outbreaks ( $\geq 7$ patients) as determined by multiple logistic regression analysis with stepwise variable selection

\begin{tabular}{|c|c|c|c|c|}
\hline Risk factor & \# all NO & Odds ratio & $95 \%$ confidence interval & p-value \\
\hline \multicolumn{5}{|l|}{ departments } \\
\hline surgery & 76 & 0.42 & $0.19-0.92$ & 0.030 \\
\hline gynecology & 20 & 6.89 & $1.55-30.63$ & 0.011 \\
\hline pediatrics & 9 & 0.05 & $0.00-0.45$ & 0.008 \\
\hline \multicolumn{5}{|l|}{ occurrence after 1989} \\
\hline reference value: no & 71 & 1.00 & n. d. & 0.024 \\
\hline yes & 72 & 0.48 & $0.22-1.06$ & 0.068 \\
\hline \multicolumn{5}{|l|}{ aware of carrier status } \\
\hline reference value: no & 116 & 1.00 & n. d. & 0.024 \\
\hline yes & 14 & 0.11 & $0.02-0.55$ & 0.008 \\
\hline \multicolumn{5}{|l|}{ transmission } \\
\hline unknown & 13 & 0.16 & $0.03-0.75$ & 0.020 \\
\hline
\end{tabular}

$N O=$ nosocomial outbreak; $n$. $d$. = not defined.

(by $89 \%$ ) of causing a LO. In the last 20 years there was some tendency towards smaller outbreaks.

\section{Discussion}

NO in surgery

Most of all included NO caused by staff happened in surgical departments (76 of $150 \mathrm{NO}$ ), but the univariate and the multiple regression analysis specified these outbreaks to be significantly smaller than the other NO. Surgeons may extraordinary strong focus on nosocomial infections, because those represent the most frequent complication in their medical field. Thus, spreaders may be detected earlier which then will lead to smaller NO. The operating theatre was the location of transmission in $80 \%$ (61 of $76 \mathrm{NO}$ ) of all NO in surgery. An explanation may be that there is a very short distance between head and hands of the surgeon and the patient, who is rather vulnerable during invasive procedures. 142 of 411 infections transmitted during operations (130 HBV, 10 HCV and 2 HIV) were blood borne. Surgeons often suffer from small undetected excoriations or injuries caused by sharp bone fragments, needles and other instruments. Transmission may occur through minimal lesions in gloves [4]. Harpaz et al. [5] described an NO where a surgeon infected 19 patients with $\mathrm{HBV}$, but the exact route of transmission remained unclear. He had performed an adequate operating technique and no obvious breaches in hygiene could be discovered. Other common agents were gram positive bacteria like Streptococcus Group A (121 infections) or S. aureus (101 infections). These agents caused mostly SSI (87.8\%; 195 of 222 infections).

\section{NO in gynecology}

$\mathrm{NO}$ in gynecology are not only very common (20 NO; thereof 11 NO in obstetrics), they are also significantly larger $(\mathrm{OR}=6.89)$ than $\mathrm{NO}$ in other medical departments (Table 2). In 7 NO a gynecologist transmitted $\mathrm{HBV}$ to a patient during an operation. And in the remaining 2 NO kitchen workers contaminated food with enteritic Salmonella and infected patients in the whole hospital. In obstetrics the most common agent was $S$. aureus (5 of $11 \mathrm{NO}$ ), which was being transmitted to neonates and parturient. There were also other agents transmitted via contact (Streptococcus group A in $4 \mathrm{NO}$ and $S$. marcescens in $1 \mathrm{NO}$ ). During a delivery the women giving birth have close and prolonged contact with e.g. midwifes (4 NO), which facilitates to transmit agents via contact. Dave et al. [6] reported 2 NO where midwifes transmitted S. aureus to 12 and 10 parturients and caused staphylococcal scalded skin syndrome (SSSS). The latest NO in obstetrics was an $\mathrm{NO}$ in France described by Occelli et al. [7] where an auxiliary nurse was nasal colonized with $S$. aureus and caused bullous impetigo in 7 neonates and colonization in 3 further neonates. Perhaps the tendency to early discharging of patients after birth may contribute to the problem of large NO in gynecology, because infections may occur only after discharge and therefore NO maybe detected later.

\section{HCW characteristics}

The only significant detail we know about the spreading $\mathrm{HCW}$ is that those HCW who were aware of their positive carrier status caused smaller outbreaks than the median (Tables 1 and 2). There were $14 \mathrm{HCW}$ that caused NO, although they knew of their infectivity, 12 physicians, 1 nurse and 1 acupuncturist. Thereof 10 employees who had a viral hepatitis (9 HBV, $1 \mathrm{HCV})$.

Being a carrier and knowing so may improve compliance to general infection control measures and by this 
lower the risk of pathogen spread. This result also suggests the presumption that $\mathrm{HCW}$ pay more attention to a possible NO, if they know of their risk to infect their patients. They detect NO earlier and therefore can terminate it earlier with less affected patients.

There is also the possibility that HCW denied awareness of their carrier status, because they could fear of jobrelated and judicial consequences, but a viral hepatitis is an infection that is probably more difficult to deny. In addition, viral hepatitis represents an infection, which is not easily transmitted and so NO may have been smaller. There is a tendency of blood borne infections to smaller $\mathrm{NO}$, which is with a $\mathrm{p}=0.57$ nearly significant (Table 1 ).

$\mathrm{HCW}$ that were only colonized and not infected caused rather large NO $(58.9 \%)$ than others $(43.0 \%)(\mathrm{p}=0.54)$ (Table 1). Only $1.4 \%$ of the colonized HCW were aware of their carrier status, compared to $17.1 \%$ of infected $\mathrm{HCW}$. $\mathrm{HCW}$ with more than 5 years of work experience caused LO just as often as HCW with 5 or less years of work experience did (Table 1). Experience of HCW does not necessarily provide significant more safety in patient care. In fact, there is a tendency of becoming less thorough in the compliance to recommendations [8].

Figure 2 shows that physicians caused most NO in absolute numbers (59 of $144 \mathrm{NO}$ ). Compared to the rather small proportion of physicians at all hospital staff, one may wonder why this group of HCW has likely been involved in NO. One explanation may be, that physicians have often and intensive contact, for example during invasive procedures, where the patient is rather vulnerable. Nearly all NO caused by physicians (72.9\%; 43 of $59 \mathrm{NO})$ were transmitted in the operating theatre. NO caused by physicians may also get publicized more frequently than $\mathrm{NO}$ due to other HCW. Finally we cannot exclude the possibility of inadequate $\mathrm{HH}$ by physicians [9].

\section{General limitations}

The most important limitation distorting these results is the publication bias. Presumably NO will have been detected more often than they get published. Supposedly those NO, that have been published have more often been of certain interest or came up with significant results.

Furthermore we could only include those outbreaks where the route of transmission could clearly be found out. Therefore easily transmittable agents like Norovirus and others are surely underrepresented. Many hospitals may have restrained from publishing their NO because of fear of bad reputation.

Another limitation is incomplete information in articles. For example we cannot provide data on the extent of cooperation between ward staff and infection control personnel, microbiologists, occupational health physicians or public health colleagues, but its quality often has a significant impact on both the prevention and the management of NO. There is also a lack of data on most socio-economic factors and/or underlying diseases of the patients that may predispose for a nosocomial infection. Especially information concerning the spreading $\mathrm{HCW}$ (e.g., on his HBV vaccination status) was scarce and complicated this analysis. The limit of 7 patients (= median) for $\mathrm{LO}$ is rather high, but reflects that chances of publication outrange chances of outbreak detection.

\section{Conclusion}

Practical consequences. Screening of personnel should not be performed regularly, as less than $10 \%$ of NO are caused by HCW. However, if certain species of microorganisms (e.g. S. aureus, HBV, S. pyogenes) are involved, the possibility of a carrier should be taken into account.

\section{Additional file}

Additional file 1: Complete list of included articles.

\section{Competing interests}

The authors herewith declare that there are no competing interests.

\section{Authors' contributions}

LD was responsible for collecting and interpreting of the data on the nosocomial outbreaks. PG has made substantial contributions to conception and design and revised the manuscript critically. FS performed statistical analyses. RPV cross-checked data extraction, participated in the conception an design of the study and primarily drafted the manuscript. All authors read and approved the final manuscript.

\section{Acknowledgement}

The authors would like to thank the Deutsche Forschergemeinschaft (DFG) for sponsorship of the Open Access Publication process.

\section{Author details}

${ }^{1}$ Institute for Medical Microbiology and Hospital Epidemiology, Hannover Medical School, Carl-Neuberg-Str. 1, Hannover D-30625, Germany. ${ }^{2}$ Institute for Hygiene and Environmental Health, Charité - University Medicine Berlin, Berlin, Germany.

Received: 21 June 2012 Accepted: 20 February 2013

Published: 22 February 2013

\section{References}

1. Vonberg RP, Weitzel-Kage D, Behnke M, Gastmeier P: Worldwide outbreak database: the largest collection of nosocomial outbreaks. Infection 2011, 39:29-34.

2. Centers for Disease Control (CDC): Recommendations for protection against viral hepatitis. MMWR Morb Mortal Wkly Rep 1985, 34:313-335.

3. Centers for Disease Control (CDC): Guidelines for prevention of transmission of human immunodeficiency virus and hepatitis B virus to health-care and public-safety workers. MMWR Morb Mortal Wkly Rep 1989, 38(Suppl 6):1-37.

4. Adams S, Stojkovic SG, Leveson SH: Needlestick injuries during surgical procedures: a multidisciplinary online study. Occup Med (Lond) 2010, 60:139-144.

5. Harpaz R, Von Seidlein L, Averhoff FM, Tormey MP, Sinha SD, Kotsopoulou K, Lambert SB, Robertson BH, Cherry JD, Shapiro CN: Transmission of hepatitis $B$ virus to multiple patients from a surgeon without evidence of inadequate infection control. N Engl J Med 1996, 334:549-554.

6. Dave J, Reith S, Nash JQ, Marples RR, Dulake C: A double outbreak of exfoliative toxin-producing strains of Staphylococcus aureus in a maternity unit. Epidemiol Infect 1994, 112:103-114. 
7. Occelli P, Blanie M, Sanchez R, Vigier D, Dauwalder O, Darwiche A, Provenzano B, Dumartin C, Parneix P, Venier AG: Outbreak of staphylococcal bullous impetigo in a maternity ward linked to an asymptomatic healthcare worker. J Hosp Infect 2007, 67:264-270.

8. Graf K, Chaberny IF, Vonberg RP: Beliefs about hand hygiene: a survey in medical students in their first clinical year. Am J Infect Control 2011. 39:885-888

9. Pittet D, Hugonnet S, Harbarth S, Mourouga P, Sauvan V, Touveneau S, Perneger TV: Effectiveness of a hospital-wide programme to improve compliance with hand hygiene. Infection control programme. Lancet 2000, 356:1307-1312.

doi:10.1186/1471-2334-13-98

Cite this article as: Danzmann et al:: Health care workers causing large nosocomial outbreaks: a systematic review. BMC Infectious Diseases 2013 13:98.

\section{Submit your next manuscript to BioMed Central and take full advantage of:}

- Convenient online submission

- Thorough peer review

- No space constraints or color figure charges

- Immediate publication on acceptance

- Inclusion in PubMed, CAS, Scopus and Google Scholar

- Research which is freely available for redistribution 